\title{
An oncolytic herpes simplex virus vector, G47D, synergizes with paclitaxel in the treatment of breast cancer
}

\author{
WEI-GEN ZENG ${ }^{1}$, JUN-JIE LI ${ }^{2}$, PAN HU ${ }^{1}$, LAN LEI ${ }^{1}$, JIA-NI WANG $^{1}$ and REN-BIN LIU ${ }^{1}$ \\ ${ }^{1}$ Breast Cancer Center, The Third Affiliated Hospital of Sun Yat-sen University, Guangzhou, Guangdong 510630; \\ ${ }^{2}$ Department of Breast Surgery, The Sichuan Province Cancer Hospital, Chengdu, Sichuan 610041, P.R. China
}

Received December 17, 2012; Accepted February 14, 2013

DOI: $10.3892 /$ or.2013.2359

\begin{abstract}
Paclitaxel-containing treatment regimens are standard chemotherapy schemes for breast cancer patients. The use of oncolytic herpes simplex virus (oHSV) vectors has been shown to be a safe and effective therapeutic approach for different types of cancer. We hypothesized that paclitaxel in combination with an oHSV vector would present an enhanced killing effect when used against breast cancer cells. In the present study, we demonstrated that the combined use of the oHSV vector G47 $\Delta$ and paclitaxel produced a synergistic effect against breast cancer cells both in vitro and in vivo. In vitro studies demonstrated that paclitaxel and G47 $\Delta$ both caused dose-dependent cytotoxicity against the human breast cancer cell lines MCF-7 and MDA-MB-468. G47D and paclitaxel also demonstrated synergistic cytotoxicity when applied together, with Chou-Talalay combination indices ranging from 0.44 to 0.77 for MCF-7 cells and 0.68 to 0.83 for MDA-MB-468 cells. Paclitaxel did not enhance viral replication or viral spread among tumor cells. However, G47 $\Delta$ increased the antitumor ability of paclitaxel by inducing mitotic arrest and apoptosis. In vivo studies indicated that when combined with $\mathrm{G} 47 \Delta$, the dose of paclitaxel could be reduced at least 5-fold while maintaining levels of tumor reduction similar to those achieved with the administration of paclitaxel alone. Combination therapy resulted in no morbidity in vivo. Our data demonstrated that G47 $\Delta$ and paclitaxel combination therapy had synergistic effects in the treatment of breast cancer. This combination therapy may be promising for breast cancer patients.
\end{abstract}

\section{Introduction}

Breast cancer is the most frequently diagnosed cancer and the leading cause of cancer-related mortality in females (1). With

Correspondence to: Professor Ren-Bin Liu, Breast Cancer Center, The Third Affiliated Hospital of Sun Yat-sen University, Guangzhou, Guangdong 510630, P.R. China

E-mail: liur@vip.163.com

Key words: breast cancer, oncolytic virus, herpes simplex virus, paclitaxel, combination therapy the development of gene-expression profiling, researchers have found that breast cancer can be divided into different molecular subtypes with distinct clinical features and varying responses to therapeutic regimens (2-4). Patients with luminal A-type tumors may gain little benefit from chemotherapy. For those patients, hormone therapy may be sufficient for systemic treatment (5). However, chemotherapy remains the standard systemic treatment for other types of cancer, particularly human epidermal growth factor receptor 2 (HER-2)-positive tumors and basal-like tumors. Paclitaxel-containing drug regimens are the standard chemotherapy schemes for the treatment of breast cancer. Relative to anthracycline, paclitaxel significantly improves the disease-free survival and overall survival of patients; however, paclitaxel has a greater effect when used in conjunction with anthracycline (6-9).

The use of oncolytic viruses is a relatively new strategy in cancer therapy. Oncolytic herpes simplex virus (oHSV) vectors are inherently cytotoxic to and specific for tumor cells (10). The vector $\mathrm{G} 47 \Delta$ was constructed as a third-generation replicationcompetent HSV-1 vector from HSV-1 laboratory strain F. The ICP47 gene and both copies of the $\gamma 34.5$ neurovirulence gene are deleted in $\mathrm{G} 47 \Delta$, and the ribonucleotide reductase (RR) gene is inactivated by insertion of the E. coli LacZ gene (11). Due to these gene deletions/mutations, the replication ability of the virus is attenuated and, therefore, the safety and tumor selectivity are increased. The use of G47 $\Delta$ has been shown to be a safe and effective therapeutic approach for various types of cancer (11-14).

Several preclinical studies have demonstrated that when oHSVs are used in combination with various cytotoxic agents, increased treatment efficacy for various malignancies is observed (15-18). GADD34 is a DNA repair enzyme that is homologous with the HSV-1 $\gamma 34.5$ gene. Due to safety considerations, $\gamma 34.5$ was deleted in the construction of G47D. This deletion attenuates the replication ability of HSV. Previous studies have demonstrated that chemotherapy agents that upregulate GADD34 in cancer cells in response to DNA damage enhance the antitumor effects of oHSV by facilitating viral proliferation (15-18). However, no published studies have investigated the mechanistic interactions between G47 $\Delta$ and paclitaxel in the treatment of breast cancer.

Our previous preclinical studies showed that G47 $\Delta$ can effectively treat primary breast tumors and brain and lung metastases $(14,19,20)$. In the present study, we found that G47 $\Delta$ 
and paclitaxel, when administered together, demonstrated a synergistic effect against the breast cancer cell lines MCF-7 and MDA-MB-468 in vitro. However, the use of paclitaxel did not enhance or impair viral proliferation. G47 $\Delta$ replicated and spread among cancer cells, causing cancer cell lysis and enhancing paclitaxel activity. G47 $\Delta$ facilitated the induction of mitotic arrest and apoptosis by paclitaxel. Finally, in vivo efficacy studies demonstrated that G47 $\Delta$ and paclitaxel combination therapies are a safe and effective regimen for the treatment of breast cancer. When using paclitaxel in combination with G47 $\Delta$, the dose of paclitaxel could be reduced at least 5 -fold while maintaining levels of tumor reduction similar to those achieved with the administration of paclitaxel alone. To the best of our knowledge, this is the first study to demonstrate that the combination of G47 $\Delta$ and paclitaxel has synergistic effects in the treatment of breast cancer.

\section{Materials and methods}

Cells and viruses. MCF-7 and MDA-MB-468 cells (all obtained from Dr Xiao-Ming Xie, Sun-sen University Cancer Center, China) were grown in Dulbecco's modified Eagle's medium (DMEM; Invitrogen, Carlsbad, CA, USA) supplemented with $10 \%$ heat-inactivated fetal calf serum (Invitrogen) and $4.5 \mathrm{~g} / 1$ glucose. Vero cells (African green monkey kidney cells; American Type Culture Collection, Manassas, VA, USA) were grown in DMEM supplemented with $10 \%$ heat-inactivated fetal calf serum (Invitrogen). Cells were cultured at $37^{\circ} \mathrm{C}$ and $5 \% \mathrm{CO}_{2}$. G47 $\Delta$ was obtained from Samuel D. Rabkin (Molecular Neurosurgery Laboratory, Massachusetts General Hospital, Harvard Medical School, Boston, MA, USA) and it was constructed as previously described (11) .

Cell susceptibility assays and Chou-Talalay analysis. MCF-7 and MDA-MB-468 cells were seeded at 2,000 cells/well into 96-well plates. Following overnight incubation, paclitaxel (Sigma, St. Louis, MO, USA) or G47 $\Delta$ was added at the indicated concentration. To select the appropriate dose ranges, we used serial dilutions to test cell susceptibility. After 4 days of incubation, cytotoxicity assays were performed using a Cell Counting Kit-8 (Dojindo, Japan) according to the manufacturer's instructions. Median effect doses $\left(E_{50}\right)$ were calculated for the drug and G47 $\Delta$ for each cell line.

For combination studies, G47 $\Delta$ and paclitaxel were added to cells at a fixed dose ratio, and Cell Counting Kit- 8 assays were performed after 4 days of incubation. To analyze the combination of G47 $\Delta$ and paclitaxel, Chou-Talalay combination indices $(\mathrm{CI})$ were calculated using CompuSyn software (Combo Syn, Inc., Paramus, NJ, USA). Fixed ratios of G47ム and paclitaxel and mutually exclusive equations were used to determine the CIs. A CI between 0.9 and 1.1 is considered additive, whereas $\mathrm{CI}<0.9$ and $\mathrm{CI}>1.1$ indicate synergism and antagonism, respectively.

Viral proliferation. To determine whether paclitaxel enhances G47 $\Delta$ proliferation, we tested the viral titers with or without the addition of paclitaxel. MCF-7 and MDA-MB-468 cells were seeded into 12 -well plates at $2 \times 10^{4}$ cells/well overnight and then treated with G47 $\Delta$ at an MOI of 0.5 (MCF-7) or 1.5
(MDA-MB-468) and with paclitaxel at $1 \mathrm{nmol} / \mathrm{l}$ (MCF-7) or $3 \mathrm{nmol} / \mathrm{l}$ (MDA-MB-468). The cells and supernatants were collected for 4 days. After three freeze-thaw cycles, the titers of infectious virus were determined using a plaque assay with Vero cells. Next, we calculated the viral pfu per viable cancer cell. The cells were treated with G47 $\Delta$ and a range of paclitaxel doses for 2 days. The viable cells were counted, and the viral titers were calculated.

Flow cytometric analysis of the cell cycle. MCF-7 and MDA-MB-468 cells were seeded into $10-\mathrm{cm}$ dishes at $8 \times 10^{5}$ cells/plate and treated with either the mock treatment, $\mathrm{G} 47 \Delta$, paclitaxel or the combination of G47 $\Delta$ and paclitaxel. At the indicated time points, the adherent and detached cells were collected and fixed in $70 \%$ ethanol at $4^{\circ}$ overnight. The cells were then washed twice in phosphate-buffered saline (PBS) containing bovine serum albumin $(0.5 \%)$ and treated in $1 \mathrm{ml}$ of PBS containing $0.1 \%$ Triton-X, RNase A $(100 \mu \mathrm{g} / \mathrm{ml}$; Sigma) and propidium iodide (PI) $(50 \mu \mathrm{g} / \mathrm{ml}$; Sigma) at room temperature for $30 \mathrm{~min}$. The cells were then immediately analyzed by flow cytometry in a BD FACSCalibur. The resulting data were analyzed with ModFit LT v3.2 (Verity Software House, Topsham, ME, USA).

Apoptosis assay. MCF-7 and MDA-MB-468 cells were seeded into $6-\mathrm{cm}$ dishes at $4 \times 10^{5}$ cells/plate and treated with either the

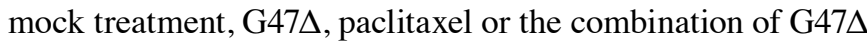
and paclitaxel. Following $48 \mathrm{~h}$ of incubation, the adherent and detached cells were collected and washed twice with PBS. The cells were then counted, adjusted to a density of $1 \times 10^{6} / \mathrm{ml}$, and double-stained with fluorescein isothiocyanate-conjugated Annexin-V and PI using an Annexin-V-FITC Apoptosis Detection Kit according to the manufacturer's instructions (Nanjing KeyGen Biotech., Co., Ltd., Nanjing, China). The stained cells were then immediately analyzed using flow cytometry with a BD FACSCalibur. The resulting data were analyzed using FlowJo v8.5.3 software (Tree Star, Ashland, OR, USA).

In vivo treatment studies. First, $5 \times 10^{6} \mathrm{MDA}-\mathrm{MB}-468$ cells were implanted into the left flank of 6-week-old female BALB/c nude mice (Vital River Laboratory Animal Technology Co., Ltd., Beijing, China). When the maximum diameters of the tumors reached $\sim 5 \mathrm{~mm}$, the mice were randomized into 5 groups $(n=7$ per group): a $\mathrm{G} 47 \Delta$ treatment group treated with intratumor

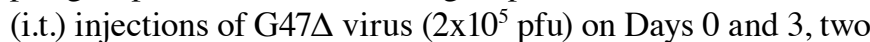
paclitaxel treatment groups treated with intraperitoneal (i.p.) injections of paclitaxel ( 3 or $15 \mathrm{mg} / \mathrm{kg}$ ) on Days 0 and 7 of a 7-day cycle for 2 weeks, a combination treatment group treated with G47 $\left(2 \times 10^{5} \mathrm{pfu}\right)$ and paclitaxel $(3 \mathrm{mg} / \mathrm{kg})$, and a mock treatment group. The tumor volume was calculated using the formula width $(\mathrm{mm})^{2} \mathrm{x}$ length $(\mathrm{mm}) \mathrm{x} 0.5$. The body weight and motor activity of each animal were monitored as indicators of general health and toxicity.

Statistical analysis. The Student's t-test (two-tailed) was used to analyze the significance of differences between the treatment groups. The test was implemented using the SPSS version 13.0 software. A P-value of $<0.05$ was considered to indicate a statistically significant difference. 
A

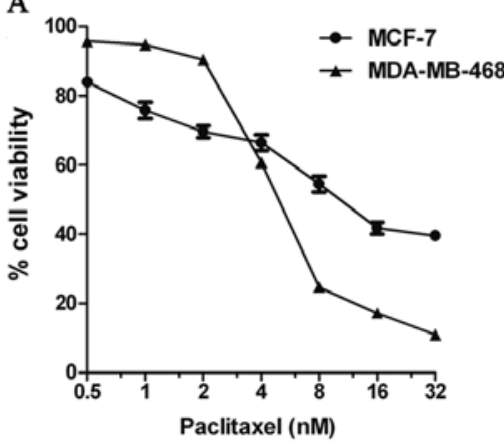

B

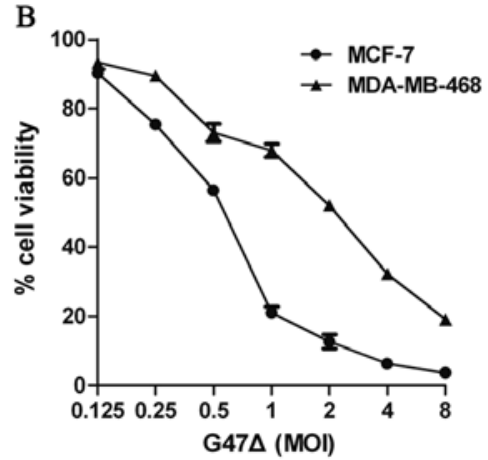

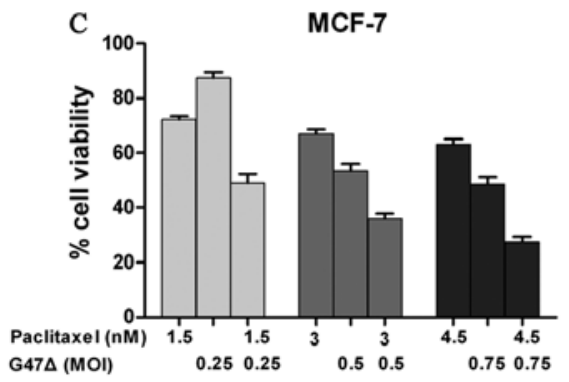
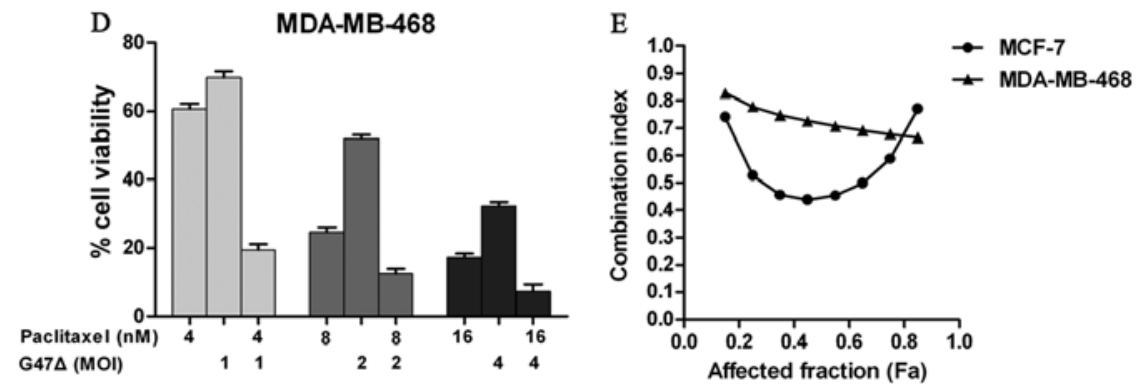

Figure 1. G47 $\Delta$ and paclitaxel exhibit a synergistic killing effect against MCF-7 and MDA-MB-468 cells in vitro. (A-D) MCF-7 and MDA-MB-468 cells were mock-treated or treated with a range of concentrations of paclitaxel or G47 $\Delta$ alone or in combination for 4 days. Cytotoxicity was evaluated using Cell Counting Kit-8 assays. The CIs of G47 $\Delta$ in combination with paclitaxel were calculated using the Chou-Talalay analyses. (E) Fraction affected (Fa) vs. combination index plots are shown. A CI $<0.9$ indicates synergy, a CI between 0.9 and 1.1 indicates addictive effects, and a $\mathrm{CI}$ of $>1.1$ indicates antagonism. These data are presented as the means \pm SD from three independent experiments.

\section{Results}

G47A exhibits a synergistic effect in conjunction with paclitaxel in the killing of breast cancer cells in vitro. First, we performed cytotoxicity assays of paclitaxel and G47D with MCF-7 and MDA-MB-468 cells. Both the MCF-7 and MDA-MB-468 cells exhibited dose-dependent cytotoxicity following exposure to paclitaxel or G47 (Fig. 1). MDA-MB-468 cells were more sensitive than MCF-7 cells to paclitaxel at higher concentrations (4-32 nmol/l), although MDA-MB-468 cells were less sensitive at lower concentrations $(0.5-2 \mathrm{nmol} / \mathrm{l})$. MCF-7 cells were more sensitive than MDA-MB-468 cells to G47 $\Delta$ cytotoxicity. The $\mathrm{ED}_{50}$, i.e., the dose causing $50 \%$ cytotoxicity, was calculated for each agent. The $\mathrm{ED}_{50}$ values of $\mathrm{G} 47 \Delta$ were an MOI of 0.70 (MCF-7) and an MOI of 1.93 (MDA-MB-468). The $\mathrm{ED}_{50}$ values of paclitaxel were $11.54 \mathrm{~nm} / \mathrm{l}(\mathrm{MCF}-7)$ and $5.90 \mathrm{~nm} / 1$ (MDA-MB-468).

Next, we performed combination cytotoxicity assays at a fixed concentration ratio based on the above results. CIs were calculated to determine whether G47D and paclitaxel used in combination exhibit a synergetic breast cancer cellkilling effect in vitro. As defined, a CI between 0.9 and 1.1 is considered additive, whereas $\mathrm{CI}<0.9$ and $\mathrm{CI}>1.1$ indicate synergism and antagonism, respectively. The combination of G47 $\Delta$ and paclitaxel produced a synergistic effect against both MCF-7 and MDA-MB-468 cells (Fig. 1). The Chou-Talalay CIs ranged from 0.44 to 0.77 for MCF-7 cells and from 0.68 to 0.83 for MDA-MB-468 cells. The most significant synergistic effect was achieved at moderate and high concentrations for MCF-7 cells and MDA-MB-468 cells, respectively. Overall, these results demonstrate that $\mathrm{G} 47 \Delta$ and paclitaxel exhibit a synergistic anti-MCF-7 and anti-MDA-MB-468 effect in vitro when used in combination.

Paclitaxel does not affect the replication of G474. To determine whether paclitaxel affects the replication of G47D in vitro, we calculated viral titers using plaque assays after infecting the cells with G47 $\Delta$ and exposing the cells to paclitaxel or vehicle. The viral titers were tested daily. We found that paclitaxel did not significantly affect the replication of G47 (Fig. 2A and B). To extend these results, we next tested viral proliferation using different doses of paclitaxel and calculated the viral titer per viable cell. Similar to our previous results, the viral titers per viable cell did not significantly change across the different paclitaxel doses tested against MCF-7 or MDA-MB-468 cells (Fig. 2C and D). Finally, X-gal staining of MCF-7 cells infected with G47 $\Delta$ and exposed to different doses of paclitaxel confirmed that paclitaxel did not significantly affect the spread of G47 (Fig. 2E).

G474 facilitates the induction of mitotic arrest by paclitaxel. Subsequently, we determined whether G47 $\Delta$ affects the antitumoral activity of paclitaxel. Paclitaxel exerts its cytotoxic effect by interacting with $\beta$-tubulin, stabilizing the structure of microtubules and preventing the depolymerization of microtubules. This microtubule stabilization leads to cell cycle arrest in the G2/M phase and ultimately leads to cell death by apoptosis. We found that G47 $\Delta$ alone did not significantly influence the cell cycle (Fig. 3). However, relative to treatment with paclitaxel alone, treatment with G47 $\Delta$ plus paclitaxel significantly promoted cell cycle arrest in the G2/M phase after a 24-h exposure for both MCF-7 and MDA-MB-468 cells (Fig. 3). 

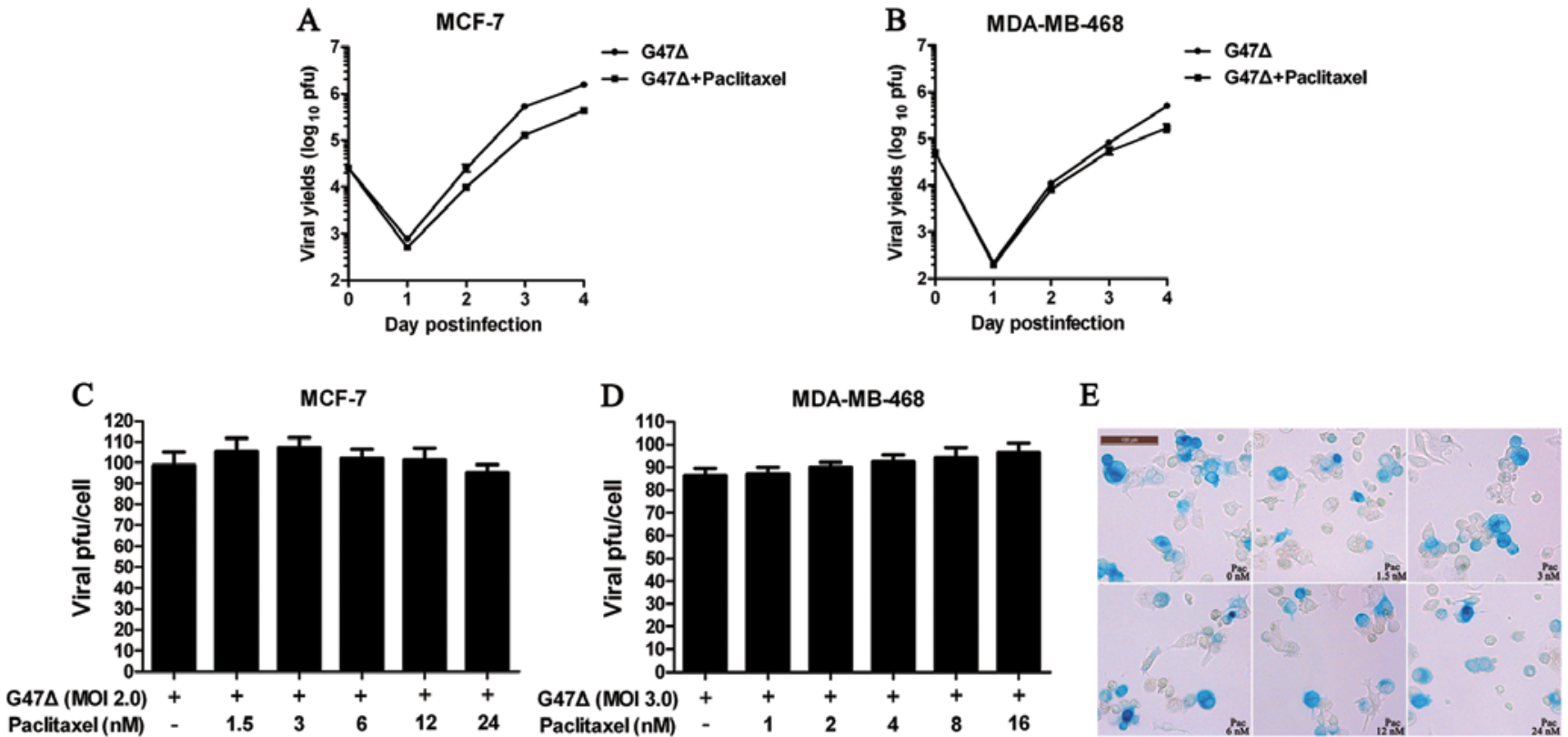

Figure 2. Paclitaxel does not enhance or attenuate the replication of G47 $\Delta$. Viral proliferation was measured after exposure of cells to G47 at an MOI of 0.5 (MCF-7) or 1.5 (MDA-MB-468) in combination with paclitaxel at $1 \mathrm{nmol} / 1$ (MCF-7) or $3 \mathrm{nmol} / 1$ (MDA-MB-468). (A and B) Viral titers were calculated daily using plaque assays. Paclitaxel slightly impaired viral proliferation in MCF-7 cells and had no influence in MDA-MB-468 cells. Next, the viral pfu per viable cell after $48 \mathrm{~h}$ of exposure of the cells to G47 $\Delta$ alone or in combination with paclitaxel was measured. (C and D) Paclitaxel did not significantly affect viral proliferation in either the MCF-7 or the MDA-MB-468 cells. X-gal staining after $48 \mathrm{~h}$ of infection yielded similar results. The data are presented as the means \pm SD from three independent experiments. (E) Scale bar, $100 \mu \mathrm{m}$.
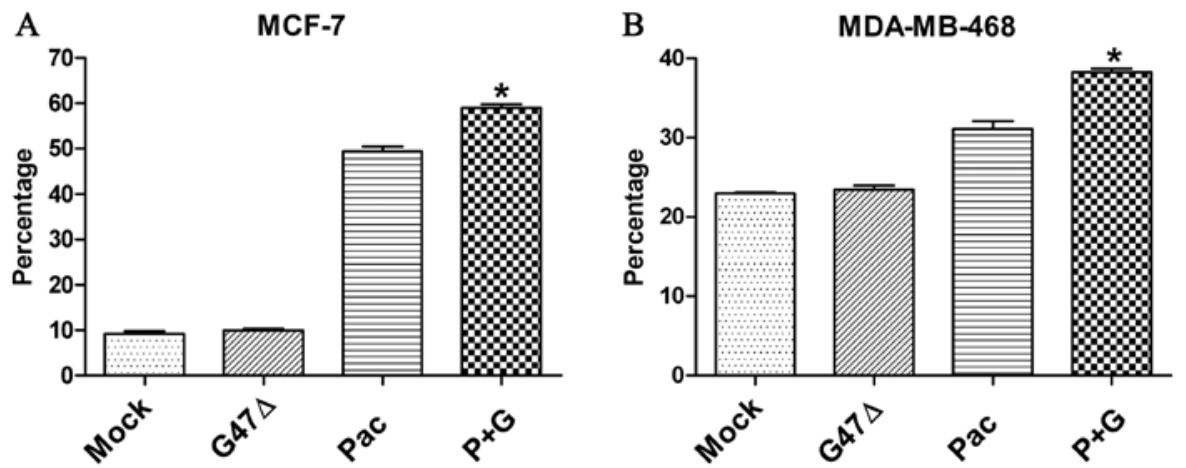

Figure 3. G47 $\Delta$ facilitates the induction of mitotic arrest by paclitaxel. The percentage of G2/M phase cells was calculated by flow cytometry after $24 \mathrm{~h}$ of exposure to mock treatment, G47 $\Delta$ at an MOI of 1 (MCF-7) or 1.5 (MDA-MB-468), paclitaxel (pac) at $6 \mathrm{nmol} / 1$ (MCF-7 and MDA-MB-468) or a combination of the vector and the drug $(\mathrm{P}+\mathrm{G})$. G47 $\Delta$ alone did not induce mitotic arrest in either cell line, but the use of paclitaxel in combination with G47 $\Delta$ resulted in a significantly higher percentage of cells in mitotic arrest than the other treatments did. The data shown represent the means \pm SD from three independent experiments. ${ }^{*} \mathrm{P}<0.05$.

For MCF-7 cells, the percentage of G2/M phase-arrested cells for the paclitaxel alone group and the combination group were $49.35 \pm 1.01 \%$ and $58.96 \pm 0.83 \%(\mathrm{P}<0.001)$, respectively. For MDA-MB-468 cells, the percentages were $31.11 \pm 0.96 \%$ and $38.29 \pm 0.43 \%(\mathrm{P}<0.001)$, respectively.

G47A enhances the ability of paclitaxel to induce apoptosis in breast cancer cells. We used two methods to assess the level of apoptosis. First, we calculated the percentage of sub-G0 cells after $72 \mathrm{~h}$ of exposure to the mock treatment, paclitaxel, $\mathrm{G} 47 \Delta$ or a combination of paclitaxel and G47 . Relative to the mock treatment $(1.17 \pm 0.12 \%)$, paclitaxel alone $(15.70 \pm 0.95 \%)$, G47 $\Delta$ alone $(5.37 \pm 0.385 \%)$, and the combination of paclitaxel and G47 $(24.29 \pm 0.60 \%)$ significantly increased the level of apoptosis in MCF-7 cells ( $\mathrm{P}<0.05$ for all comparisons) (Fig. 4A). Similar results were observed with MDA-MB-468 cells (Fig. 4B). Annexin-V and PI staining also confirmed that paclitaxel and $\mathrm{G} 47 \Delta$ in combination produced a significant increase in apoptosis (Fig. 4C).

G474 and paclitaxel exhibit synergistic effects in vivo. Finally, we tested whether G47 $\triangle$ and paclitaxel could exhibit a synergistic effect in vivo. We chose MDA-MB-468 cells to form tumors in BALB/C mice. After flank tumors developed, the mice were divided into 5 treatment groups. One group was treated with a mock treatment, another group received 
A

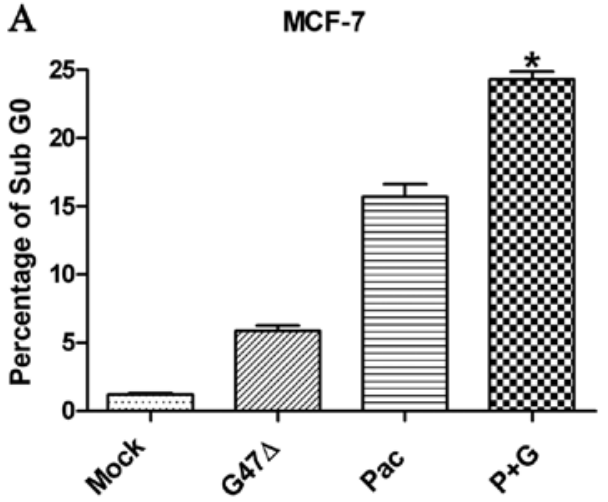

$\mathrm{C}$

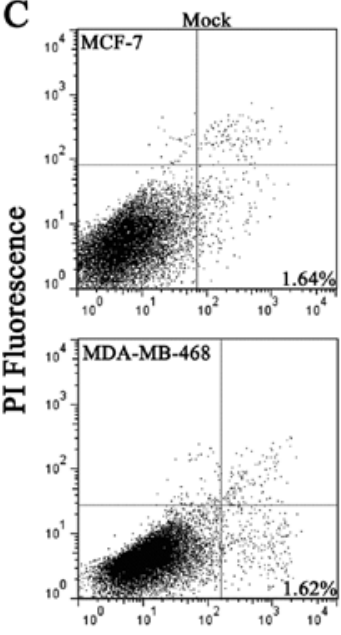

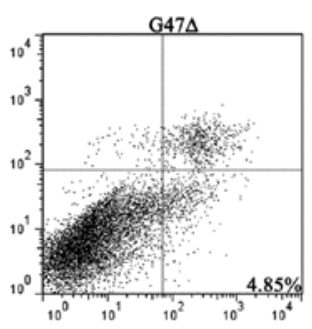

B $\quad$ MDA-MB-468
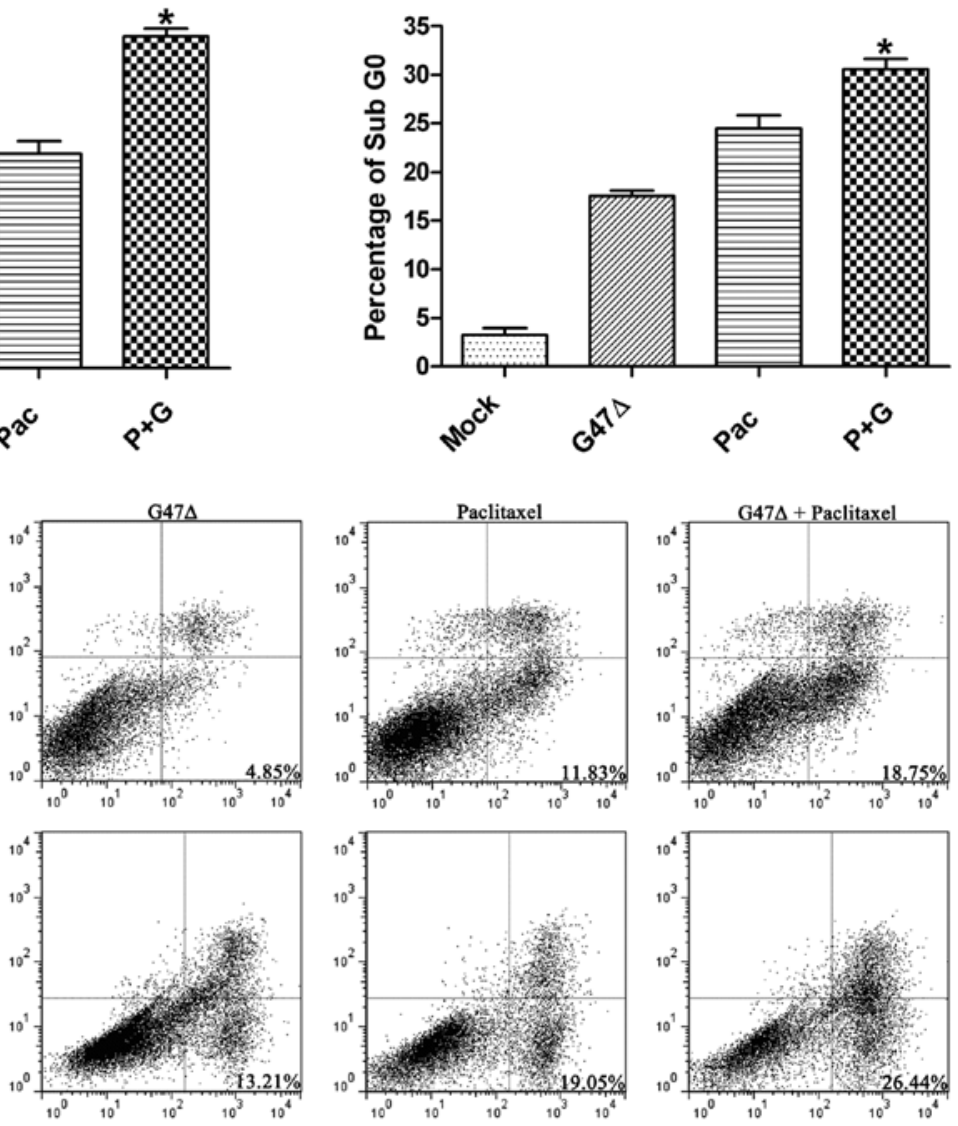

Annexin-V Fluorescence

Figure 4. G47 $\Delta$ enhances the induction of breast cancer cell apoptosis by paclitaxel. (A and B) After $72 \mathrm{~h}$ of exposure to the mock treatment, G47 $\Delta$ at an MOI of 1 (MCF-7) or 1.5 (MDA-MB-468), paclitaxel (pac) at $6 \mathrm{nmol} / \mathrm{l}$ (MCF-7 and MDA-MB-468) or a combination of the vector and the drug (P+G), the percentage of sub-G0 cells was calculated. Both G47D and paclitaxel induced the apoptosis of MCF-7 and MDA-MB-468 cells. However, the combination treatment resulted in a significantly greater percentage of apoptotic cells than either paclitaxel or G47D alone. We also used Annexin-V and PI staining assay to evaluate apoptosis. After $48 \mathrm{~h}$ of exposure to the mock treatment, G47 $\Delta$ at an MOI of 1 (MCF-7) or 1.5 (MDA-MB-468), paclitaxel (pac) at $6 \mathrm{nmol} / \mathrm{l}$ (MCF-7 and MDA-MB-468) or the combination of the vector and the drug (P+G), the percentage of apoptotic cells was measured. Annexin-V and PI staining also confirmed that paclitaxel and G47 $\Delta$ together produced a significant increase in apoptosis. The data shown represent the means \pm SD from three independent experiments. ${ }^{*} \mathrm{P}<0.05$.

i.t. G47 $\left(2 \times 10^{5} \mathrm{pfu}\right)$ injections on Days 0 and 3, two other groups received i.p. paclitaxel injections at different doses $(3$ or $15 \mathrm{mg} / \mathrm{kg}$ ) twice a week for 2 weeks, and one group received a combination of $\mathrm{G} 47 \Delta\left(2 \times 10^{5} \mathrm{pfu}\right)+\operatorname{paclitaxel}(3 \mathrm{mg} / \mathrm{kg})$. We found that both $\mathrm{G} 47 \Delta$ and paclitaxel could inhibit tumor growth in vivo. Compared with the mock treatment group, both the $\mathrm{G} 47 \Delta$ and paclitaxel ( $3 \mathrm{mg} / \mathrm{kg}$ ) alone groups presented a significant reduction in the mean tumor volume by Day 35 . In addition, we observed that the treatment of mice with a combination of G47 $\Delta$ and paclitaxel resulted in a synergistic effect in vivo and a significant reduction in the mean tumor volume $\left(68.71 \pm 22.65 \mathrm{~mm}^{3}\right)$ compared to treatment with $\mathrm{G} 47 \Delta$ alone $\left(277.57 \pm 75.35 \mathrm{~mm}^{3}\right)$, paclitaxel alone $\left(501.86 \pm 74.79 \mathrm{~mm}^{3}\right)$, or the mock treatment $\left(809.14 \pm 102.58 \mathrm{~mm}^{3}\right)$ by Day $35(\mathrm{P}<0.05$ for all three comparisons) (Fig. 5A). Of note, when paclitaxel was combined with $\mathrm{G} 47 \Delta$, the dose of paclitaxel could be reduced at least 5-fold while maintaining levels of tumor reduction similar to those attained with the administration of paclitaxel alone at $15 \mathrm{mg} / \mathrm{kg}$. We also found that the combination treatment produced a significant reduction in tumor weight compared with the other treatments (Fig. 5B). Furthermore, the combined treatment caused no additional signs of toxicity, and the body weight of the mice was similar among all groups (mean body weight, $22.5 \mathrm{~g}$ ). In addition, the body weight did not change significantly over the course of the study.

\section{Discussion}

Breast carcinoma is a relatively low-malignancy type of tumor. Patients who suffer from early-stage breast cancer have positive clinical outcomes. However, approximately $30 \%$ of patients with early-stage disease eventually develop recurrent or metastatic lesions (21). At present, metastatic cancer remains an incurable disease. Therefore, new therapies are urgently required. Our group demonstrated previously that G47D effectively targets primary breast tumors and brain and lung metastases $(14,19,20)$.

To increase safety and tumor selectivity, some genes were deleted from or mutated in the viral vector, including the RR gene and the $\gamma 34.5$ neurovirulence gene. These changes attenuated the replication ability of HSV, resulting in oHSV being relatively selective for dividing tumor cells as there are cellular 

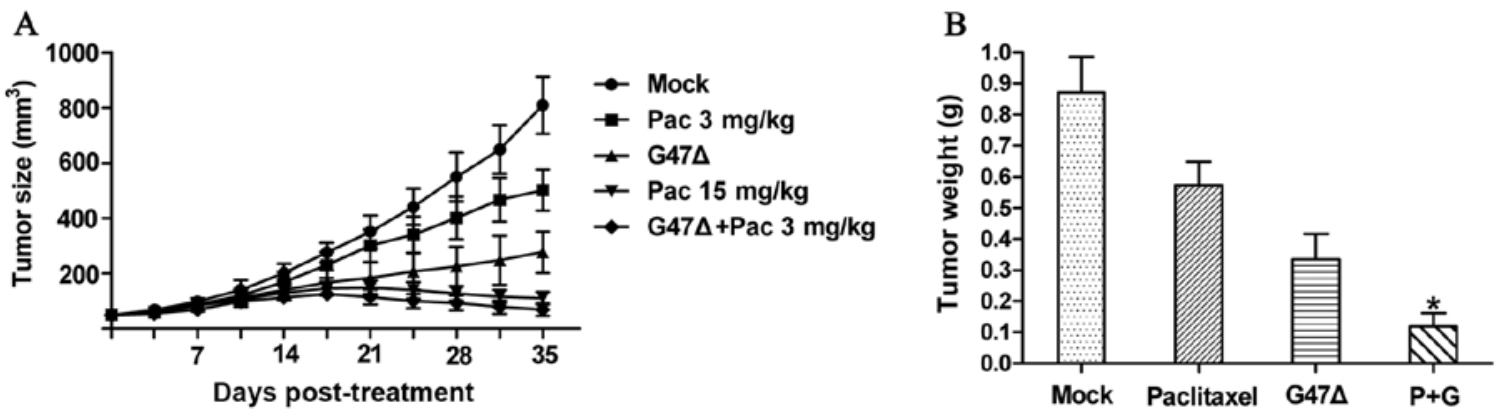

Figure 5. G47 $\Delta$ and paclitaxel have a synergistic effect in vivo. When maximum diameters of the tumors reached $\sim 5 \mathrm{~mm}$, the mice were randomized into 5 groups ( $\mathrm{n}=7$ per group): a G47 $\Delta$ treatment group treated with i.t. injections of G47 $\Delta$ virus $\left(2 \times 10^{5} \mathrm{pfu}\right)$ on Days 0 and 3 , two paclitaxel treatment groups treated with i.p. injections of paclitaxel ( 3 or $15 \mathrm{mg} / \mathrm{kg}$ ) on Days 0 and 7 of a 7-day cycle for 2 weeks, a combination treatment group treated with G47 $\left(2 \times 10^{5}\right.$ pfu) and paclitaxel $(3 \mathrm{mg} / \mathrm{kg}$ ) and a mock treatment group. (A) The combination group had significantly lower mean tumor volumes than the other three groups by Day 35. Moreover, when paclitaxel was combined with G47 , the dose of paclitaxel could be reduced 5-fold while maintaining the effectiveness of $15 \mathrm{mg} / \mathrm{kg}$ paclitaxel alone. (B) The combination group also had a significantly lower tumor weight than the other groups by Day 35 . The data shown represent the means \pm SD. ${ }^{*} \mathrm{P}<0.05$.

homologs that can compensate for the missing genes, such as RR and GADD34 for $\gamma 34.5$. Some preclinical studies have reported that oHSVs, when combined with chemotherapeutic agents that upregulate the expression or activities of RR and/ or GADD34 in tumor cells, exhibit synergistic effects against various tumors (15-18). Petrowsky et al (15) reported that fluorodeoxyuridine, which causes nucleotide pool imbalances and DNA damage-induced upregulation of RR and GADD34 in colorectal cancer cell lines, promotes enhanced viral replication and tumor cell death.

In the present study, we demonstrated, using Chou-Talalay assays, that G47 $\mathrm{A}$ and paclitaxel in combination exhibited a synergistic effect against MCF-7 and MDA-MB-468 breast cancer cells. We initially hypothesized that paclitaxel might enhance viral replication. However, both viral titer experiments and X-gal staining indicated that paclitaxel did not significantly influence viral replication or spread. These results indicated that the mechanism mediating the synergistic cytotoxicity between G47 $\Delta$ and paclitaxel does not involve the enhancement of viral replication by paclitaxel.

To further investigate the interaction between G47 $\Delta$ and paclitaxel, we determined whether G47 $\Delta$ enhanced the antitumor activity of paclitaxel. Paclitaxel stabilizes the structure of microtubules, leading to cell cycle arrest in the G2/M phase and ultimately inducing cell death by apoptosis. First, we found that although $\mathrm{G} 47 \Delta$ alone did not significantly impact the cell cycle of either MCF-7 or MDA-MB-468 cells, G47 $\Delta$ and paclitaxel, when administered in combination, resulted in significantly greater numbers of tumor cells arrested in the G2/M phase, compared to treatment with either paclitaxel or G47 $\Delta$ alone. Then, we found that both G47 $\Delta$ and paclitaxel could cause tumor cell apoptosis, but G47 $\Delta$ alone only produced a small percentage of apoptotic cells. However, G47 $\Delta$ significantly enhanced the ability of paclitaxel to induce apoptosis, relative to paclitaxel alone. Elliott and O'Hare (22) found that the HSV-1 tegument protein VP22 can reorganize microtubules into thick bundles that became highly resistant to microtubule-depolymerizing agents, suggesting that VP22 might have the capacity to stabilize the microtubule network. Our data indicate that G47 $\Delta$ administered by itself at a low dose did not significantly influence the cell cycle of MCF-7 or
MDA-MD-468 cells, but VP22 might enhance the ability of paclitaxel to stabilize the structure of microtubules, eventually leading to G2/M phase arrest and cell apoptosis.

Although paclitaxel is used widely to treat a variety of tumors, the toxic effect of the drug is a key factor in restricting its broader clinical use. Hematopoietic toxicity and cumulative peripheral neuropathy limit the long-term use of paclitaxel, which always leads to dose reduction and the delay of paclitaxel chemotherapy, particularly for patients with recurrent or metastatic disease, most of whom have been heavily treated for the primary disease. Kim et al (23) found that when breast cancer patients received adriamycin, cyclophosphamide and paclitaxel as adjuvant chemotherapy for their primary tumors, the drug dose was reduced for $17.1 \%$ of patients, and $14.3 \%$ patients delayed treatment due to the toxicity of the chemotherapy agents. Loibl et al (24) observed that patients receiving a lower relative total dose had a shorter overall survival. In the present study, we demonstrated that G47 $\Delta$ and paclitaxel combination therapy can reduce the required paclitaxel dose by at least 5 -fold while maintaining levels of tumor reduction similar to those achieved with the administration of paclitaxel alone. In addition, the combination therapy caused no additional signs of toxicity. These data may have important clinical implications. For some patients, especially patients who have ever been heavily treated for recurrent or metastatic disease, G47 $\Delta$ and paclitaxel combined therapy may be an effective and safe therapeutic regimen.

In summary, we report that a third-generation replicationcompetent HSV-1 vector G47 $\Delta$ and paclitaxel, when given in combination, had a synergistic anti-breast cancer effect both in vitro and in vivo. Paclitaxel did not significantly influence

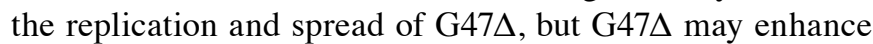
the antitumor activity of paclitaxel through mitosis arrest and apoptosis. G47 $\Delta$ and paclitaxel combined therapy appears to be an effective and safe therapeutic regimen for the treatment of breast cancer, and, thus, the presented data may have critical clinical implications for the treatment of breast cancer. To the best of our knowledge, this is the first report demonstrating a synergetic effect of a combination of G47 $\Delta$ and paclitaxel against breast cancer cells. 


\section{Acknowledgements}

This study was supported by the National Natural Science Foundation of China (grant no. 30672410) and the Natural Science Foundation of Guangdong Province, China (grant no. 06104599).

\section{References}

1. Jemal A, Bray F, Center MM, Ferlay J, Ward E and Forman D: Global cancer statistics. CA Cancer J Clin 61: 69-90, 2011.

2. Perou CM, Sørlie T, Eisen MB, et al: Molecular portraits of human breast tumours. Nature 406: 747-752, 2000.

3. Sørlie T, Perou CM, Tibshirani R, et al: Gene expression patterns of breast carcinomas distinguish tumor subclasses with clinical implications. Proc Natl Acad Sci USA 98: 1086910874, 2001.

4. Sotiriou C, Neo SY, McShane LM, et al: Breast cancer classification and prognosis based on gene expression profiles from a population-based study. Proc Natl Acad Sci USA 100: 10393-10398, 2003.

5. Lim E and Winer EP: Adjuvant chemotherapy in luminal breast cancers. Breast 20 (Suppl 3): S128-S131, 2011.

6. Paridaens R, Biganzoli L, Bruning P, et al: Paclitaxel versus doxorubicin as first-line single-agent chemotherapy for metastatic breast cancer: a European Organization for Research and Treatment of Cancer Randomized Study with cross-over. J Clin Oncol 18: 724-733, 2000.

7. Sledge GW, Neuberg D, Bernardo P, Ingle JN, Martino S, Rowinsky EK and Wood WC: Phase III trial of doxorubicin, paclitaxel, and the combination of doxorubicin and paclitaxel as front-line chemotherapy for metastatic breast cancer: an intergroup trial (E1193). J Clin Oncol 21: 588-592, 2003.

8. Ghersi D, Wilcken $\mathrm{N}$ and Simes RJ: A systematic review of taxane-containing regimens for metastatic breast cancer. Br J Cancer 93: 293-301, 2005.

9. Henderson IC, Berry DA, Demetri GD, et al: Improved outcomes from adding sequential paclitaxel but not from escalating doxorubicin dose in an adjuvant chemotherapy regimen for patients with node-positive primary breast cancer. J Clin Oncol 21: 976-983, 2003.

10. Varghese S and Rabkin SD: Oncolytic herpes simplex virus vectors for cancer virotherapy. Cancer Gene Ther 9: 967-978, 2002.

11. Todo T, Martuza RL, Rabkin SD and Johnson PA: Oncolytic herpes simplex virus vector with enhanced MHC class I presentation and tumor cell killing. Proc Natl Acad Sci USA 98 6396-6401, 2001.
12. Fukuhara $\mathrm{H}$, Martuza RL, Rabkin SD, Ito $\mathrm{Y}$ and Todo $\mathrm{T}$ : Oncolytic herpes simplex virus vector g47delta in combination with androgen ablation for the treatment of human prostate adenocarcinoma. Clin Cancer Res 11: 7886-7890, 2005.

13. Wang JN, Hu P, Zeng MS and Liu RB: Anti-tumor effect of oncolytic herpes simplex virus G47delta on human nasopharyngeal carcinoma. Chin J Cancer 30: 831-841, 2011.

14. Liu R, Varghese S and Rabkin SD: Oncolytic herpes simplex virus vector therapy of breast cancer in C3(1)/SV40 T-antigen transgenic mice. Cancer Res 65: 1532-1540, 2005.

15. Petrowsky H, Roberts GD, Kooby DA, et al: Functional interaction between fluorodeoxyuridine-induced cellular alterations and replication of a ribonucleotide reductase-negative herpes simplex virus. J Virol 75: 7050-7058, 2001.

16. Eisenberg DP, Adusumilli PS, Hendershott KJ, et al: 5-fluorouracil and gemcitabine potentiate the efficacy of oncolytic herpes viral gene therapy in the treatment of pancreatic cancer. J Gastrointest Surg 9: 1068-1077, 2005.

17. Bennett JJ, Adusumilli P, Petrowsky $\mathrm{H}$, et al: Up-regulation of GADD34 mediates the synergistic anticancer activity of mitomycin $\mathrm{C}$ and a gamma134.5 deleted oncolytic herpes virus (G207). FASEB J 18: 1001-1003, 2004.

18. Aghi M, Rabkin S and Martuza RL: Effect of chemotherapyinduced DNA repair on oncolytic herpes simplex viral replication. J Natl Cancer Inst 98: 38-50, 2006.

19. Liu R, Martuza RL and Rabkin SD: Intracarotid delivery of oncolytic HSV vector G47Delta to metastatic breast cancer in the brain. Gene Ther 12: 647-654, 2005.

20. Wang J, Hu P, Zeng M, Rabkin SD and Liu R: Oncolytic herpes simplex virus treatment of metastatic breast cancer. Int J Oncol 40: 757-763, 2012.

21. Gonzalez-Angulo AM, Morales-Vasquez F and Hortobagyi GN: Overview of resistance to systemic therapy in patients with breast cancer. Adv Exp Med Biol 608: 1-22, 2007.

22. Elliott G and O'Hare P: Herpes simplex virus type 1 tegument protein VP22 induces the stabilization and hyperacetylation of microtubules. J Virol 72: 6448-6455, 1998.

23. Kim WY, Woo SU, Seo JH, Son GS, Lee JB and Bae JW: Toxicities, dose reduction and delay of docetaxel and paclitaxel chemotherapy in breast cancer without distant metastases. J Cancer Res Ther 7: 412-415, 2011.

24. Loibl S, Skacel T, Nekljudova V, et al: Evaluating the impact of Relative Total Dose Intensity (RTDI) on patients' short and long-term outcome in taxane- and anthracycline-based chemotherapy of metastatic breast cancer- a pooled analysis. BMC Cancer 12: 121-131, 2011. 